\title{
Index rerum ad Vol. 178
}

\section{Confecit: C. Loeb-Schürch}

$(\mathrm{V})=$ Proceedings - Verhandlungsberichte -

Congrès $(\mathrm{B})=$ Book Reviews - Buchbesprechungen -

Livres nouveaux

Abflussfazilität, v. TRH

Ablatio, v. Prophylaxie, Retinal detachment

surgery, Retinal function, Transconjunctival

buckling Abrasio corneae, v. Weichlinse Acide chlorhydrique, v. Burning Acide

vanilmandélique, v. Vanilmandelic acid Acuité visuelle, v. Albinism, Doppler encephalique, Gyrate atrophy, Retinal function, Rétino-

blastomes Addison's disease, v. General medicine Adenoma; benign adenoma of the ciliary epithelium with tumour seeding 67 Aderhaut, v. Gyrate atrophy, Naevus Adrenalin, v.

Therapeutic trial, Timolol Albinism 19

Alder's anomaly, v. Gyrate atrophy Aldosteronism, v. General medicine Alport's syndrome, v.

Lenticonus Aminoacidopathy, v. Gyrate atrophy,

Hyperglycinuria Amsler test, v. Retinal function Amyloidosis, v. Meretoja syndrome

Angiographie fluorescéinique, v. Hyphaema,

Microangiopathy, Naevus Angle camérulaire, v. Ring melanoma,

Simple glaucoma Anésthésie générale, v. Eye position Aneurysma, v. Hyphaema, Kyste osseux Anophthalmia, v. Choristoma Anterior chamber, v. Hyphaema, Ring melanoma Antibiotica, v. Rhodotorula Anticipation, v. Simple glaucoma Antigen, v. Hepatitis B Antikörper, v.

Panenzephalitis Aphakia correction in juvenile cataract 110 (V) Apparatus; a portable ERG apparatus $90(\mathrm{~V})$

Arteriosklerose, v. Doppler encephalique

Ascomyceten, v. Monosporiosis

Aspiration technique; routine bimanual aspiration

technique for double capsular pocket fixation

117 (V) Ataxie, v. Heredoataxia, Wallenberg-Syndrom Atrophia gyrata, v. Gyrate atrophy

Atrophie irienne, v. Iris atrophy Augenbinnendruck, v. Iris atrophy, Simple

glaucoma, Therapeutic trial, Timolol Augenheilkunde im Kindesalter 120 (B)

Augenkomplikationen, v. Retinal detachment

surgery, Rhodotorula Aussaat, v. Adenoma Autoimmune reaction, v. Panenzephalitis

Basedow's disease, v. TRH

Benign tumours of the bony orbit 108 (V)

Betablocker, v. Timolol

Bindehaut, v. Genetics, Molluscum contagiosum,

Urea treatment Blepharoplastik, v. Euryblepharon Bluttransfusion, v. Fibroplasie Bone cyst, v. Kyste osseux Bone dystrophy, v. Kyste osseux Book reviews 119 (B), 239 (B), 404 (B) Brûlure, v. Burning 
Buchbesprechungen 119 (B), 239 (B), 404 (B) Bulbus, v. Silicone Burning; early morphological changes of the corneal epithelium after burning with hydrochloric acid. A scanning electron microscope study 327

Calcium dobesilate, v. Microangiopathy Canthoplasty, v. Euryblepharon Carcinoma, v. Urea treatment Cardiovascular disease, v. Hyphaema Carotid; super-selective arteriography of the external carotid artery 120 (B) Cataract, v. Adenoma, Epithelioma, Gyrate atrophy, Lenticonus, Leukokorie, Tiger Cataract and cataract extraction in relation with delayed hypersensitivity to lens proteins 72 (V) Cataract; transient rosette-shaped cataract after vitrectomy $106(\mathrm{~V})$

406

Index Rerum

Cellular density of normal corneal endothelium 102 (V)

Cellular immunity in keratoplasty 93 (V)

Chamber angle, v. Ring melanoma, Simple glaucoma

Chambre antérieure, v. Hyphaema, Ring melanoma

Champ visuel, v. Doppler encephalique, Optic radiation

Chandler's syndrome, v. Iris atrophy

Chirurgie, v. Euryblepharon, Eye position, Retinal detachment surgery, Retinal function, Simple glaucoma, Tiger, Transconjunctival buckling

Chorea Huntington, v. Sakkaden

Choristoma; degenerative apparent anophthalmia with complex choristoma of orbit. A clinicopathological case report 321

Choroid, v. Gyrate atrophy, Naevus

Choroidal naevus 110 (V)

Ciliary body, v. Adenoma, Epithelioma, Ring melanoma

Circulation, v. Doppler encephalique

Clean eye medication $113(\mathrm{~V})$

Clinicopathologic case reports 67, 173, 235

Clonidine; a pharmacological study of possible central components in the regulation of intraocular pressure: influence of clonidine on intraocular pressure 89 (V)

Color vision, v. Retinal function

Complications oculaires, v. Retinal detachment surgery, Rhodotorula

Cones, v. Heredoataxia

Congenital cataract, v. Tiger

Congenital glaucoma, v. Simple glaucoma

Congrès $72-118(\mathrm{~V})$

Conjunctiva, v. Genetics, Molluscum contagiosum, Urea treatment

Conjunctivitis lignosa, v. Genetics

Conn's disease, v. General medicine

Contact lens, v. Hepatitis B, Weichlinse

Cornea, v. Burning, Genetics, Iris atrophy, Meretoja syndrome, Monosporiosis, Rhodotorula, Ring melanoma, Urea treatment, Weichlinse 
Cornea transplantations; advances and disappointments, indications and restrictions in tissuetyped cornea transplantations $97(\mathrm{~V})$

Cornea verticillata, v. Genetics

Corneal vascularization and keratoplasty 94 (V)

Corps ciliaire, v. Adenoma, Epithelioma, Ring melanoma

Corps vitré, v. Fava beans, Leukokorie,

Prophylaxie Corticosteroide, v. Rhodotorula Cristallin, v. Adenoma, Lenticonus Croissance, v.

Silicone Cromoglycate; some experiences with 2-sodium

cromoglycate (Opticrom $\left.{ }^{\circledR}\right) 84$ (V) Cryocoagulation, v. Prophylaxie, Rétinoblastomes,

Transconjunctival buckling Cytology, v. Rhodotorula

Deafness, v. Albinism, Lenticonus Décollement rétinien, v. Prophylaxie, Retinal detachment

surgery, Retinal function, Transconjunctival buckling Decreased visual acuity in alcohol abuse

82 (V) Degenerative anophthalmia, v. Choristoma Dégénérescence pigmentaire, v. Naevus

Dégénérescence rétinienne, v. Prophylaxie Dégénérescence spino-cérébellaire, v. Heredoataxia

Dermatology, v. Albinism, Genetics Determination of visual acuity with the aid of the

projection system 90 (V) Diabetes, v. Microangiopathy Diagnose, v. Vanilmandelic acid

Diagnostic problems in ocular myasthenia 81 (V) Differentialdiagnose, v. Kyste osseux,

Leukokorie Doppler encephalique; le Doppler encephalique

en ophtalmologie quotidienne 37 Drusen, v. Naevus Dyscephalic syndrome, v. Genetics

Dystrophie cornéenne, v. Iris atrophy,

Meretoja syndrome, Weichlinse Dystrophie osseuse, v. Kyste osseux Dystrophie tachetée de la cornée, v. Genetics

Echographie, v. Doppler encephalique, Leukokorie EEG, v. Panenzephalitis Ektropion, v.

Euryblepharon Electrolytes, v. General medicine Electromyography, v. Eye position Elektronen-

Mikroskopie, v. Burning, Genetics,

Rhodotorula, Thioridazine Embryology, v. Choristoma Empfindlichkeit, v. Retinal function

Endocrinology, v. General medicine, TRH Endothelium cornéen, v. Iris atrophy

Ensemencement, v. Adenoma Epithel, v. Burning Epithelioma, v. Adenoma

Index Rerum

407

Epithelioma; malignant epithelioma of the

ciliary body 190 Epithéliome spinocellulaire, v. Urea treatment ERG, v. General medicine,

Gyrate atrophy,

Heredoataxia, Thioridazine Erosio corneae recidivans, v. Weichlinse Erythrozyten, v. Fava beans

Essentielle progressive Irisatrophie, v. Iris atrophy Euryblepharon; genetic study and surgical correction of euryblepharon 396 Experimentelle Forschung, v. Burning, Hepatitis B,

Rhodotorula, Silicone, Thioridazine Eye position and electromyographic observation

of squint eyes under general anesthesia 166

Fabry's disease, v. Genetics

Facilité d'écoulement, v. TRH

Farbsehen, v. Retinal function

Fava beans; vitreoretinal haemorrhages after

ingestion of fava beans in a G-6-PD-deficient

subject 259 Fibroplasie; enquête sur la fibroplasie rétrolentale

en France 1 Fisher-Syndrom; die dissozíierte Ophthalmoplegie 
der Abduktion: hintere «internukleäre»

Ophthalmoplegie im Rahmen eines Fisher-

Syndroms 365 Fixation of an iridocapsular lens 117 (V) Fluoro-Angiographie, v. Hyphaema,

Microangio-

pathy, Naevus Francois' syndrome, v. Genetics Frühgeburt, v. Fibroplasie Fundus, v. Albinism,

Gyrate atrophy

Gefässe, v. Doppler encéphalique, Hyphaema,

Kyste osseux, Microangiopathy, Naevus General medicine; electroretinogram in

general medicine 273 Genetics, v. Albinism, Gyrate atrophy, Hyper-

glycinuria, Lenticonus, Meretoja syndrome,

Panenzephalitis, Simple glaucoma, Weichlinse Genetics; consideration on the importance of genetics in ophthalmology 381 Gesichtsfeld, v. Doppler encéphalique,

Optic radiation Glaskörper, v. Fava beans, Leukokorie, Prophylaxie Glaucoma; suspect

glaucoma 86 (V) Glaucoma simplex, v. Iris atrophy, Simple

glaucoma, Therapeutic trial, Timolol, TRH

Glaucome secondaire, v. Ring melanoma Glukose-6-Phosphat-Dehydrogenase-Mangel,

v. Fava beans Glycin, v. Hyperglycinuria Gonioscopy, v. Simple glaucoma Goniotrephination beneath a scleral flap:

a 2-year follow-up study 88 (V) Growth, v. Silicone Guanethidin, v. Therapeutic trial GuillainBarré Syndrom, v. Fisher-Syndrom Gyrate atrophy of the choroid and retina 311

Haemodialysis, v. General medicine Haemodialysis; ophthalmological aspects of

chronic intermittent haemodialysis 101 (V) Haemolytic crisis, v. Fava beans Haemorrhagie, v.

Fava beans, Hyphaema Haemorrhagische Diathese, v. Albinism Haidinger phenomenon, v.

Retinal function Hamsters with Greene melanomas 106 (V) Heart catheterization; complications in heart

catheterization and haemodialysis 118 (V) Hefepilz, v. Rhodotorula Hemianopsia; pseudo-

bitemporal hemianopsia

associated with situs inversus 80 (V) Hemianopsie, v. Optic radiation Hepatitis B; das Risiko

einer Übertragung von

Hepatitis B durch Kontaktlinsenanpassung 148 Hérédité, v. Albinism, Genetics, Gyrate atrophy, Hyperglycinuria, Lenticonus, Meretoja

syndrome, Panenzephalitis, Simple glaucoma,

Weichlinse Heredoataxia (spinocerebellar degeneration), ERG

alterations, temporal aspects 267 Heterochromie, v. Ring melanoma Histochemie, v. Albinism,

Genetics Histologie, v. Adenoma, Burning, Choristoma,

Epithelioma, Genetics, Kyste osseux,

Meretoja syndrome, Molluscum contagiosum,

Rétinoblastomes, Rhodotorula, Thioridazine,

Tiger, Urea treatment Hormonal dysfunction, v. TRH Hornhaut, v. Burning, Genetics, Iris atrophy,

Meretoja syndrome, Monosporiosis, Rhodotorula,

Ring melanoma, Urea treatment, Weichlinse Hyaluronidase, v. Genetics Hyperaminoacidurie, v.

Gyrate atrophy,

Hyperglycinuria Hypercapnia, v. General medicine

408

Index Rerum 
Hyperglycinuria; rare association of hyperglycinuria and lenticonus in two members of the same family 131

Hyphaema; vascular tufts of the pupillary border causing a spontaneous hyphaema 297

Hypophyse, v. TRH

Hypothalamus, v. TRH

Iatrogenic lesions of the endothelium during

cataract extraction $100(\mathrm{~V})$ Immunological activity to corneal antigens in

patients with corneal diseases 92 (V) Immunotherapy: ocular melanoma and

immunotherapy Ill (V) Indentator, v. Transconjunctival buckling Infection, v. Hepatitis B,

Molluscum contagiosum,

Monosporiosis, Panenzephalitis, Rhodotorula Instrument, v. Transconjunctival buckling

Interpretation of fundus fluorescein angiography

404 (B) Intraocular pressure, v. Iris atrophy, Simple

glaucoma, Therapeutic trial, Timolol Ionic changes, v. General medicine Iridography, v.

Microangiopathy Iris, v. Albinism, Hyphaema, Ring melanoma Iris atrophy; a case of unilateral essential

progressive iris atrophy $90(\mathrm{~V})$ Iris atrophy; essential progressive iris atrophy.

Report of two cases 303 Irradiation, v. Rétinoblastomes Irrigation; the Rotterdam irrigation and aspiration

system $115(\mathrm{~V})$

Jodisi, v. General medicine

Kalium, v. General medicine

Kammerwinkel, v. Ring melanoma, Simple glaucoma

Kaninchen, v. Burning, Rhodotorula, Silicone

Katarakt, v. Adenoma, Epithelioma, Gyrate atrophy, Lenticonus, Leukokorie, Tiger

Keratitis, v. Rhodotorula, Weichlinse

Keratomykose, v. Monosporiosis

Keratopathy, v. Gyrate atrophy

Keratoplasty; diagnosis and therapy of complications during post-operative treatment after

keratoplasty $96(\mathrm{~V})$

Keratoplasty in progressive mycotic keratitis 95 (V)

Keratozyten, v. Genetics

Knochenzyste, v. Kyste osseux Kontaktlinsen, v. Hepatitis B, Weichlinse Kryokoagulation, v.

Prophylaxie, Rétinoblastomes,

Transconjunctival buckling Kyste osseux; le kyste osseux anévrisant de

l'orbite 121

Lacrimal fluid, v. Burning, Hepatitis B Lapin, v. Burning, Rhodotorula, Silicone Larmes, v.

Burning, Hepatitis B Laser-interferometric determination of visual

acuity 89 (V) Leigh Syndrom, v. Sakkaden Lens, v. Adenoma, Lenticonus Lens implantation;

treatment of traumatic cataract

in children by lens implantation 114 (V) Lens implants; prevention of loss of endothelium

caused by intraocular lens implants 109 (V) Lenticonus, v. Hyperglycinuria Lenticonus; familial anterior and posterior

lenticonus 186 Leukaemia; intraocular localization of malignant 
lymphoma and leukaemia 97 (V) Leukokoria, v. Rétinoblastomes Leukokorie; echographische Differentialdiagnose

der Leukokorie im Kindesalter 215 Levure, v. Rhodotorula

Lid, v. Euryblepharon, Molluscum contagiosum Linse, v. Adenoma, Lenticonus Liquor, v.

Fisher-Syndrom Livres nouveaux 119 (B), 239 (B), 404 (B)

Macula, v. Retinal function

Macular dystrophy of cornea, v. Genetics

Malformation, v. Euryblepharon, Simple glaucoma,

Tiger Masern, v. Panenzephalitis Measles, v. Panenzephalitis Médecine générale, v. General

medicine Medikament, v. Microangiopathy, Therapeutic trial,

Timolol, Urea treatment Melanin, v. Albinism Melanoma, v. Radiosensitization, Ring

melanoma,

Urea treatment Melanoma of the cornea 95 (V) Melanoma; some results of experiments with radioactive quinoline derivates in hamsters with

a melanoma 106 (V) Melanoma vs. naevus 96 (V) Melleril@, v. Thioridazine

Index Rerum

409

Membrana pupillaris persistens, v. Tiger

Meretoja syndrome. Lattice dystrophy of the cornea with hereditary generalized amyloidosis 173

Metabolism, v. Albinism, Fava beans, Genetics, Gyrate atrophy, Hyperglycinuria, Vanilmandelic acid

Methode, v. Euryblepharon, Eye position, Fibroplasie, Naevus, Prophylaxie, Retinal detachment surgery, Rétinoblastomes, Transconjunctival buckling, Weichlinse

Microangiopathy; fluorescence iris angiography in the assessment of calcium dobesilate therapy in the treatment of diabetic microangiopathy 250

Microscopie électronique, v. Burning, Genetics, Rhodotorula, Thioridazine

Microstrabismus; spontaneous recovery in micro-strabismus 79 (V)

Miotica, v. Simple glaucoma

Missbildung, v. Euryblepharon, Simple glaucoma, Tiger

Molluscum contagiosum; epidemiology of ophthal-mological complications in molluscum

contagiosum and the importance of cytodiagnosis in this condition 101 (V)

Molluscum contagiosum of the palpebral conjunctiva. Report of a case 137

Monosporiosis; corneal monosporiosis. A review and report of 1 case 142

Motor fusion through non-foveal stimuli 81 (V)

Motorik, v. Eye position, Fisher-Syndrom, Sak-kaden, Wallenberg-Syndrom

Mouvements oculaires, v. Eye position, Fisher-Syndrom, Sakkaden, Wallenberg-Syndrom

Movements of the eyes 120 (B)

Mucopolysaccharide, v. Genetics

Multiple Sklerose, v. General medicine

Mykose, v. Monosporiosis, Rhodotorula

Myopie, v. Gyrate atrophy

Myotonic dystrophy, v. General medicine

Naevus of the choroid 156

Narkose, v. Eye position

Natrium, v. General medicine 
Netherlands Ophthalmological Society, 172nd annual congress, Groningen 1978 72-118 (V) Netzhaut, v. Doppler encéphalique, Fava beans, Gyrate atrophy, Panenzephalitis, Prophylaxie, Retinal function, Rétinoblastomes, Thioridazine

Neuritis, v. Fisher-Syndrom

Neuroblastom, v. Vanilmandelic acid

Neuro-Ophthalmologie, v. Doppler encéphalique, Fisher-Syndrom, General medicine, Heredoataxia, Optic radiation, Panenzephalitis, Sakkaden, TRH, Wallenberg-Syndrom Neuro-ophthalmology update 239 (B)

Non-ruptural retinal detachment after complicated and uncomplicated lens extractions 103 (V) Nystagmography as a diagnostic tool in multiple sclerosis 85 (V)

Nystagmus, v. Fisher-Syndrom, Wallenberg-Syndrom

Objective automatic refraction $88(\mathrm{~V})$

Ocular movements, v. Eye position, Fisher-Syndrom, Sakkaden, Wallenberg-Syndrom

Oculographie, v. Fisher-Syndrom, Sakkaden, Wallenberg-Syndrom

Oculopharyngeal dystrophy: diagnostic possibilities and problems 74 (V)

Operation, v. Euryblepharon, Eye position, Retinal detachment surgery, Retinal function, Simple

glaucoma, Tiger, Transconjunctival buckling

Ophthalmoplegia-plus 74 (V)

Ophthalmoplegie, v. Fisher-Syndrom

Optic nerve atrophy in Surinam 83 (V)

Optic radiation; videopupillographic and VER investigations in patients with congenital and acquired lesions of the optic radiation 348

Orbita, v. Choristoma, Kyste osseux, Silicone

Ornithine, v. Gyrate atrophy

Orthoptics; comprehensive review of orthoptics and ocular motility 119 (B)

Orthoptie pratique 239 (B)

Oscillatory potential, v. Thioridazine

Oxygénothérapie, v. Fibroplasie

Paedo-Ophthalmologie, v. Fibroplasie, Leukokorie, Panenzephalitis, Rétinoblastomes, Simple glaucoma, Vanilmandelic acid

Panenzephalitis; Augenbefunde bei der subakut sklerosierenden Panenzephalitis 337

Papillophlebitis 84 (V)

Paralysie supranucléaire progressive, v. Sakkaden

Paresis of the trochlear nerve $80(\mathrm{~V})$

Parkinsonism, v. General medicine

Pathogenität, v. Rhodotorula

410

Index Rerum

Pattern-evoked cortical potentials in children, without and with movie-pictures 87 (V) Pattern-evoked cortical potentials in multiple slerosis 87 (V) Paupière, v. Euryblepharon, Molluscum contagiosum Périmétrie statique, v. Optic radiation, Retinal function Perimetry; principles of quantitative perimetry: 
testing and interpreting the visual field 119 (B) Photocoagulation, v. Prophylaxie,

Rétinoblastomes Photography, v. Naevus Photomydriasis 92 (V) Pigment cells, v. Albinism, Naevus, Radio-

sensitization, Ring melanoma Pinealoblastoma with photoreceptor differentiation

94 (V) Pineal tumors 119 (B)

Plasmocytoma; epibulbar plasmocytoma 115 (V) Plattenepithel-Karzinom, v. Urea treatment Polyneuritis, v. Fisher-Syndrom Porenzephalie, v. Optic radiation Position des yeux, v. Eye position Potassium, v. General medicine Potentiel oscillatoire, v. Thioridazine Premature, v. Fibroplasie Proceedings 72-118 (V) Progressive Irisatrophie, v. Iris atrophy Progressive supranukleäre Parese, v. Sakkaden Prophylaxie du décollement rétinien 12 Pseudogliom, v. Leukokorie Pseudotumor, v. Kyste osseux Psychopharmaca, v. Thioridazine Pupillarmembran, v. Tiger Pupille, v. Hyphaema

Rabbit, v. Burning, Rhodotorula, Silicone

Radiation optique, v. Optic radiation

Radiographie, v. Kyste osseux

Radiosensitization; clinical trials on chemical radiosensitization of malignant melanomas of the choroid 194

Radiotherapie, v. Radiosensitization, Rétinoblastomes

Rat, v. Thioridazine

Recherches expérimentales, v. Burning, Hepatitis B, Rhodotorula, Silicone, Thioridazine

Retina, v. Doppler encéphalique, Fava beans, Gyrate atrophy, Panenzephalitis, Prophylaxie,

Retinal function, Rétinoblastomes, Thioridazine

Retinal detachment, v. Prophylaxie, Retinal

detachment surgery, Retinal function, Trans-

conjunctival buckling Retinal detachment surgery; considerations upon

retinal detachment surgery 263 Retinal function after surgical treatment of

detachment 210

Retinal haemorrhages in the high mountains 78 (V)

Retinoblastoma, v. Leukokorie, Vanilmandelic acid

Rétinoblastomes; traitement des rétinoblastomes, clinique de Lausanne, 1944-1978 5

Retinoschisis; senile retinoschisis 404 (B)

Retrolentale Fibroplasie, v. Fibroplasie, Leukokorie

Rhodotorula; corneal infections by rhodotorula 241

Rieger's anomaly with retinal pigmentation 107 (V)

Ring melanoma; diffuse malignant ring melanoma of the iris and ciliary body 235

Robot nurse; demonstration of a robot operating-room nurse 103 (V)

Rougeole, v. Panenzephalitis

Rubeosis iridis, v. Microangiopathy

Sakkade, v. Fisher-Syndrom, Wallenberg-

Syndrom Sakkaden; verlangsamte Sakkaden bei

verschiedenen neurologischen Erkrankungen

357 Salzsäure, v. Burning Sarcoidosis; an unusual localization of sarcoidosis

108 (V) Säureverätzung, v. Burning Scheie procedure; a 4-year follow-up on the

double-flap Scheie procedure 87 (V) Schielen, v. Eye position, Rétinoblastomes Sclérose en plaques, v. General medicine Seeding, v. Adenoma Sehschärfe, v. Albinism, Doppler

encéphalique, 
Gyrate atrophy, Retinal function, Rétinoblastomes Sehstrahlung, v. Optic radiation Sekundärglaukom, v. Ring melanoma Sensitivity, v. Retinal function Serum proteins; the presence of serum proteins and crystallins in bovine aqueous humour 100

(V)

Index Rerum

411

Silicone; adult rabbit eye and orbital volumes after periodic intrabulbar injections of silicone 43 Simple glaucoma before the age of 30 years 32 SI units in ophthalmological practice 84 (V) Sklerosierende Panenzephalitis, v. Panenzephalitis Slow-Virus-Infektion, v. Panenzephalitis Sodium, v. General medicine Spatial aspects of orbital musculo-fibrous tissue in man 119(B) Spinocerebellar degeneration, v. Heredoataxia Spontaneous carotico-cavernous fistulae: the importance of the typically arterialized aspect of the anterior conjunctival veins and secondary glaucoma in the diagnosis and conservative therapy of this condition 73 (V) Squamous cell carcinoma, v. Urea treatment Squint, v. Eye position, Rétinoblastomes Static perimetry, v. Optic radiation, Retinal function Steinert's myotonic dystrophy, v. General medicine Stenose, v. Doppler encéphalique Steroide, v. Rhodotorula Strabismus, v. Eye position, Rétinoblastomes Strabismus in monozygotic twins 73 (V) Subakut sklerosierende

Panenzephalitis,

v. Panenzephalitis Subretinal fluid, v. Retinal detachment surgery Surdité, v. Albinism, Lenticonus Surgery, v. Euryblepharon, Eye position, Retinal detachment surgery, Retinal function, Simple glaucoma, Tiger, Transconjunctival buckling Synapse, v. General medicine Syndrom, v. Albinism, Fisher-Syndrom, Genetics, Iris atrophy, Lenticonus, Meretoja syndrome, Wallenberg-Syndrom Synechie, v. Iris atrophy

Tapetum lucidum, v. Tiger

Taubheit, v. Albinism, Lenticonus

Tension oculaire, v. Iris atrophy, Simple glaucoma, Therapeutic trial, Timolol

Teratom, v. Choristoma

Therapeutic trial of the combination in an eye lotion of adrenaline $(0,5$ or $1 \%)$ and guanethidine ( 3 or $5 \%$ ) in cases of open-angle glaucoma 49

Therapie, v. Fibroplasie, Kyste osseux, Micro-angiopathy, Radiosensitization, Rétinoblastomes, Rhodotorula, Simple glaucoma, Timolol, Urea treatment, Weichlinse Thioridazine; change in oscillatory potential by thioridazine 220 Thyreoidea, v. General medicine, TRH Tiger; Operation einer kongenitalen Katarakt bei einem sibirischen Tiger 56 Timolol; klinische Erfahrungen mit Timololaugentropfen 204 Tobacco amblyopia: effect of therapy on the electroretinogram 73 (V) Tomographie, v. Kyste osseux Tomography; the diagnostic limitations of

computerized axial tomography 120 (B) Tonography, v. TRH Torticollis; a case of periodic alternating torticollis, treated by retro-equatorial myopexy 76(V) Toxizität, v. Thioridazine Toxoplasmose, v. Panenzephalitis Toxoplasmosis; a prospective study of toxo- 
plasmosis and pregnancy, with a follow-up on

infected children 105 (V) Trabeculopuncture; experience with laser

trabeculopuncture 89 (V) Tränenflüssigkeit, v. Burning, Hepatitis B Transconjunctival buckling

for retinal detachment

16 Transfusion sanguine, v. Fibroplasie TRH; evaluation de Гaxe hypothalamo-hypophyso-

thyroïdien par la TRH dans le glaucome

chronique simple 226 Tumor, v. Adenoma, Choristoma, Epithelioma,

Kyste osseux, Molluscum contagiosum,

Naevus, Radiosensitization, Rétinoblastomes,

Ring melanoma, Urea treatment, Vanil-

mandelic acid Tyrosinase, v. Albinism

Ultrasonographie, v. Doppler encéphalique,

Leukokorie Ultrasound; surprising ultrasound 104 (V) Uraemie, v. General medicine, Lenticonus Urea treatment; effects of urea treatment in

malignancies of the conjunctiva and cornea 198 Urin, v. Hyperglycinuria, Vanilmandelic acid

Vaisseaux, v. Doppler encéphalique, Hyphaema,

Kyste osseux, Microangiopathy, Naevus Vanilmandelic acid; excretion of vanilmandelic

acid in the urine of retinoblastoma patients 181

412

Index Rerum

Varia 240, 404

VER, v. Optic radiation

Verätzung, v. Burning

Vererbung, v. Albinism, Genetics, Gyrate atrophy,

Hyperglycinuria, Lenticonus, Meretoja

syndrome, Panenzephalitis, Simple glaucoma,

Weichlinse Verhandlungsberichte 72-118 (V) Verre de contact, v. Hepatitis B, Weichlinse

Vessels, v. Doppler encephalique, Hyphaema,

Kyste osseux, Microangiopathy, Naevus Videopupillographie, v. Optic radiation Virus, v.

Hepatitis B, Molluscum contagiosum,

Panenzephalitis Vision des couleurs, v. Retinal function Visual acuity, v. Albinism, Doppler

encephalique,

Gyrate atrophy, Retinal function, Rétino-

blastomes Visual-evoked response in rivalry and suppression

in normal and abnormal binocular vision 83 (V) Visual field, v. Doppler encephalique, Optic

radiation Visually evoked response, v. Optic radiation Vitrectomy; comparative experiences with

vitrectomy instruments 104 (V)

Vitrectomy; gradually changing indications for

vitrectomy 105 (V) Vitreous body, v. Fava beans, Leukokorie,

Prophylaxie Vorderkammer, v. Hyphaema, Ring melanoma

Wachstum, v. Silicone

Waldenström's disease; manifestation of

Waldenström's disease in the palpebra 114 (V)

Wallenberg-Syndrom; systematische Abweichung sakkadischer Augenbewegungen beim

Wallenberg-Syndrom 373 
Weichlinse; die Bedeutung der therapeutischen Weichlinse in der Behandlung der rezidivierenden Erosio corneae 289

Wernicke Syndrom, v. Sakkaden

Yeast, v. Rhodotorula

Zapf en, v. Heredoataxia

Z. Ziliarkörper

v. Adenoma

Epithelioma

Ring

melanoma Zirkulation

v. Doppler encephalique Zytologie

v. Rhodotorula 